\title{
The Pediatric Hospital Medicine Core Competencies: 2020 Revision Introduction and Methodology
}

\author{
Jennifer Maniscalco, MD, MPH, MAcM, FAAP ${ }^{1,2}$, Sandra Gage, MD, PhD, SFHM, FAAP 3,4 , \\ Sofia Teferi, MD, SFHM, FAAP5 ${ }^{5}$ Erin Stucky Fisher, MD, MHM, FAAP ${ }^{6,7}$
}

\begin{abstract}
${ }^{1}$ Department of Pediatrics, Johns Hopkins University School of Medicine, Baltimore, Maryland; '2Johns Hopkins All Children's Hospital, St. Petersburg, Florida; ${ }^{3}$ Department of Child Health, University of Arizona College of Medicine - Phoenix, Phoenix, Arizona; ${ }^{4}$ Division of Hospital Medicine, Phoenix Children's Hospital, Phoenix, Arizona; ${ }^{5}$ Physician Executive, Richmond, Virginia; ${ }^{6}$ Department of Pediatrics, University of California San Diego School of Medicine, San Diego, California; ${ }^{7}$ Rady Children's Hospital San Diego, San Diego, California.
\end{abstract}

BACKGROUND: The Pediatric Hospital Medicine (PHM) Core Competencies define the expertise required of practitioners and provide a framework for educational activities. Since initial publication in 2010, the scope of practice for pediatric hospitalists has evolved in clinical, research, administrative, and educational arenas.

OBJECTIVE: To describe the methodology utilized in the revision of The PHM Core Competencies to ensure a product reflective of current roles and expectations for pediatric hospitalists across all training pathways and practice settings.

METHODS: The Society of Hospital Medicine (SHM) Pediatrics Special Interest Group supported the initiation of the revision. A diverse group of editors and authors was engaged from among members of SHM, the American Academy of Pediatrics and the Academic Pediatrics Association. Editorial roles were expanded to represent all practice settings. More than 80 individuals contributed, representing both university and community sites, and all
US geographic regions. Editors conducted a two-part needs assessment; a survey related to content was distributed to the PHM community and content from recent conferences and $\mathrm{PHM}$ related publications was reviewed. The final compendium consists of 4 sections and 66 chapters, including 12 new chapters and 36 chapters with substantial changes. Individual chapters and the entire compendium underwent rigorous internal and external review.

CONCLUSION: The PHM Core Competencies: 2020 Revision reflects the work of a broad spectrum of PHM practitioners responding to the practice and educational changes in PHM over the past decade. The compendium can inform education, training, and career development for pediatric hospitalists practicing now and in coming years. Journal of Hospital Medicine 2020;15(6):389-394. (C) 2020 Society of Hospital Medicine. Originally published online in the Pediatric Hospital Medicine Core Competencies 2020 Revision. J Hosp Med. 2020;15(Suppl1):e12-e17. rate he Pediatric Hospital Medicine Core Competencies were first published in 2010 to help define a specific body of knowledge and measurable skills needed to practice high quality care for hospitalized pediatric patients across all practice settings. ${ }^{1}$ Since then, the number of practicing pediatric hospitalists has grown to a conservative estimate of 3,000 physicians and the scope of practice among pediatric hospitalists has matured. ${ }^{2}$ Pediatric hospitalists are increasingly leading or participating in organizational and national efforts that emphasize interprofessional collaboration and the delivery of high value care to hospitalized children and their caregivers-including innovative and family-centered

Corresponding Author: Sandra Gage, MD, PhD;

Email: Sgage1@phoenixchildrens.com.

Published online June 1, 2020.

Find additional supporting information in the online version of this article.

Received: December 6, 2019; Revised: January 20, 2020;

Accepted: January 27, 2020

(C) 2020 Society of Hospital Medicine DOI 10.12788/jhm.3391 care models, patient safety and quality improvement initiatives, and research and educational enterprises. ${ }^{3-8}$ In response to these changes, the American Board of Medical Specialties designated Pediatric Hospital Medicine (PHM) as a pediatric subspecialty in 2016.

The field of PHM in the United States continues to be supported by three core societies-Society of Hospital Medicine (SHM), American Academy of Pediatrics (AAP), and Academic Pediatric Association (APA). Together, these societies serve as tri-sponsors of the annual Pediatric Hospital Medicine national conference, which now welcomes over 1,200 attendees from the United States and abroad. ${ }^{9}$ Each society also individually sponsors a variety of professional development and continuing medical education activities specific to PHM.

In addition, pediatric hospitalists often serve a pivotal role in teaching learners (medical students, residents, and other health profession students), physician colleagues, and other healthcare professionals on the hospital wards and via institutional educational programs. Nearly 50 institutions in the United States offer graduate medical education training in PHM. ${ }^{10}$ The PHM Fellowship Directors Council has developed a stan- 
dardized curricular framework and entrustable professional activities, which reflect the tenets of competency-based medical education, for use in PHM training programs. ${ }^{11-13}$

These changes in the practice environment of pediatric hospitalists, as well as the changing landscape of graduate and continuing medical education in PHM, have informed this revision of The PHM Core Competencies. The purpose of this article is to describe the methodology of the review and revision process.

\section{OVERVIEW OF THE PHM CORE COMPETENCIES: 2020 REVISION}

The PHM Core Competencies: 2020 Revision provide a framework for graduate and continuing medical education that reflects the current roles and expectations for all pediatric hospitalists in the United States. The acuity and complexity of hospitalized children, the availability of pediatric subspecialty care and other resources, and the institutional orientation towards pediatric populations vary across community, tertiary, and children's hospital settings. In order to unify the practice of PHM across these environments, The PHM Core Competencies: 2020 Revision address the fundamental and most common components of PHM which are encountered by the majority of practicing pediatric hospitalists, as opposed to an extensive review of all aspects of the field.

The compendium includes 66 chapters on both clinical and nonclinical topics, divided into four sections-Common Clinical Diagnoses and Conditions, Core Skills, Specialized Services, and Healthcare Systems: Supporting and Advancing Child Health (Table 1). Within each chapter is an introductory paragraph and learning objectives in three domains of educational outcomes_cognitive (knowledge), psychomotor (skills), and affective (attitudes) - as well as systems organization and improvement, to reflect the emphasis of PHM practice on improving healthcare systems. The objectives encompass a range of observable behaviors and other attributes, from foundational skills such as taking a history and performing a physical exam to more advanced actions such as participating in the development of care models to support the health of complex patient populations. Implicit in these objectives is the expectation that pediatric hospitalists build on experiences in medical school and residency training to attain a level of competency at the advanced levels of a developmental continuum, such as proficient, expert, or master. ${ }^{14}$

The objectives also balance specificity to the topic with a timeless quality, allowing for flexibility both as new information emerges and when applied to various educational activities and learner groups. Each chapter can stand alone, and thus themes recur if one reads the compendium in its entirety. However, in order to reflect related content among the chapters, the appendix contains a list of associated chapters (Chapter Links) for further exploration. In addition, a short reference list is provided in each chapter to reflect the literature and best practices at the time of publication.

Finally, The PHM Core Competencies: 2020 Revision reflect the status of children as a vulnerable population. Care for hos- pitalized children requires attention to many elements unique to the pediatric population. These include age-based differences in development, behavior, physiology, and prevalence of clinical conditions, the impact of acute and chronic disease states on child development, the use of medications and other medical interventions with limited investigative guidance, and the role of caregivers in decision-making and care delivery. Heightened awareness of these factors is required in the hospital setting, where diagnoses and interventions often include the use of high-risk modalities and require coordination of care across multiple providers.

\section{METHODS}

Project Initiation

Revision of The PHM Core Competencies: 2020 Revision began in early 2017 following SHM's work on The Core Competencies in Hospital Medicine 2017 Revision. ${ }^{15}$ The Executive Committee of the SHM Pediatrics Special Interest Group (SIG) supported the initiation of the revision. The 3 editors from the original compendium created an initial plan for the project that included a proposed timeline, processes for engagement of previously involved experts and new talent, and performance of a needs assessment to guide content selection. The Figure highlights these and other important steps in the revision process.

\section{Editor and Associate Editor Selection}

The above editors reviewed best practice examples of roles and responsibilities for editor and associate editor positions from relevant, leading societies and journals. From this review, the editors created an editorial structure specifically for The PHM Core Competencies: 2020 Revision. A new position of Contributing Editor was created to address the need for dedicated attention to the community site perspective and ensure review of all content, within and across chapters, by a pediatric hospitalist who is dedicated to this environment. Solicitation for additional editors and associate editors occurred via the SHM Pediatrics SIG to the wider SHM membership. The criteria for selection included active engagement in regional or national activities related to the growth and operations of PHM, strong organizational and leadership skills, including the ability to manage tasks and foster creativity, among others. In addition, a deliberate effort was made to recruit a diverse editorial cohort, considering geographic location, primary work environment, organizational affiliations, content expertise, time in practice, gender, and other factors.

\section{Chapter Topic Selection}

The editors conducted a two-pronged needs assessment related to optimal content for inclusion in The PHM Core Competencies: 2020 Revision. First, the editors reviewed content from conferences, textbooks, and handbooks specific to the field of PHM, including the conference programs for the most recent 5 years of both the annual PHM national conference and annual meetings of PHM's 3 core societies in the United States-SHM, AAP, and APA. Second, the editors conducted a needs assessment survey with several stakeholder groups, 
TABLE 1. The Pediatric Hospital Medicine Core Competencies: 2020 Revision Sections and Chapters

\begin{tabular}{|c|c|c|c|}
\hline Common Clinical Diagnoses and Conditions & Core Skills & Specialized Services & $\begin{array}{l}\text { Healthcare Systems: Supporting } \\
\text { and Advancing Child Health }\end{array}$ \\
\hline Acute Abdominal Pain and Acute Abdomen & $\begin{array}{l}\text { Bladder Catheterization and Interpretation } \\
\text { of Urinalysis }\end{array}$ & Acute Behavioral and Psychiatric Conditions & Advocacy \\
\hline Acute Gastroenteritis & Communication & Adolescent and Young Adult Medicine & Business Practices \\
\hline Acute Respiratory Failure & Diagnostic Imaging & Child Abuse and Neglect & Consultation and Co-management \\
\hline Altered Mental Status & Electrocardiogram Interpretation & Child with Medical Complexity & Education \\
\hline Asthma & Feeding Tubes & Chronic Behavioral and Psychiatric Conditions & Ethics \\
\hline Bone and Joint Infections & Intravenous Access and Phlebotomy & Newborn Care and Delivery Room Management & Evidence-based Medicine \\
\hline Brief Resolved Unexplained Event & Lumbar Puncture & Palliative Care and Hospice & Family Centered Care \\
\hline Bronchiolitis & Non-invasive Monitoring & Pediatric Interfacility Transport & Handoffs and Transitions of Care \\
\hline Central Nervous System Infections & Nutrition & & Health Information Technology \\
\hline Constipation & Oxygen Delivery and Airway Management & & High Value Care \\
\hline Diabetes Mellitus & Pain Management & & Infection Control and Antimicrobial Stewardship \\
\hline Failure to Thrive & Pediatric Advanced Life Support & & Leadership in Healthcare \\
\hline Fever of Unknown Origin & Peri-procedural Care & & Legal Issues and Risk Management \\
\hline Fluid and Electrolyte Management & Preventive Care Services & & Patient Safety \\
\hline Gastrointestinal and Digestive Disorders & Procedural Sedation & & Quality Improvement \\
\hline Head and Neck Disorders & & & Research \\
\hline
\end{tabular}

Kawasaki Disease

Neonatal Abstinence Syndrome/Neonatal Opioid

Withdrawal Syndrome

Neonatal Fever

Neonatal Jaundice

Pneumonia

Seizures

Sepsis and Shock

Sickle Cell Disease

Skin and Soft Tissue Infections

Toxin Ingestion and Exposure

Urinary Tract Infections

including SHM's Pediatrics and Medicine-Pediatrics SIGs, AAP Section on Hospital Medicine and its subcommittees, APA Hospital Medicine SIG, PHM Fellowship Directors Council, and PHM Division Directors, with encouragement to pass the survey link to others in the PHM community interested in providing input (Appendix Figure). The solicitation asked for comment on existing chapters and suggestions for new chapters. For any new chapter, respondents were asked to note the intended purpose of the chapter and the anticipated value that chapter would bring to our profession and the children and the caregivers served by pediatric hospitalists.

The entire editorial board then reviewed all of the needs assessment data and considered potential changes (additions or deletions) based on emerging trends in pediatric healthcare, the frequency, relevance, and value of the item across all environments in which pediatric hospitalists function, and the value to or impact on hospitalized children and caregivers. Almost all survey ratings and comments were either incorporated into an 


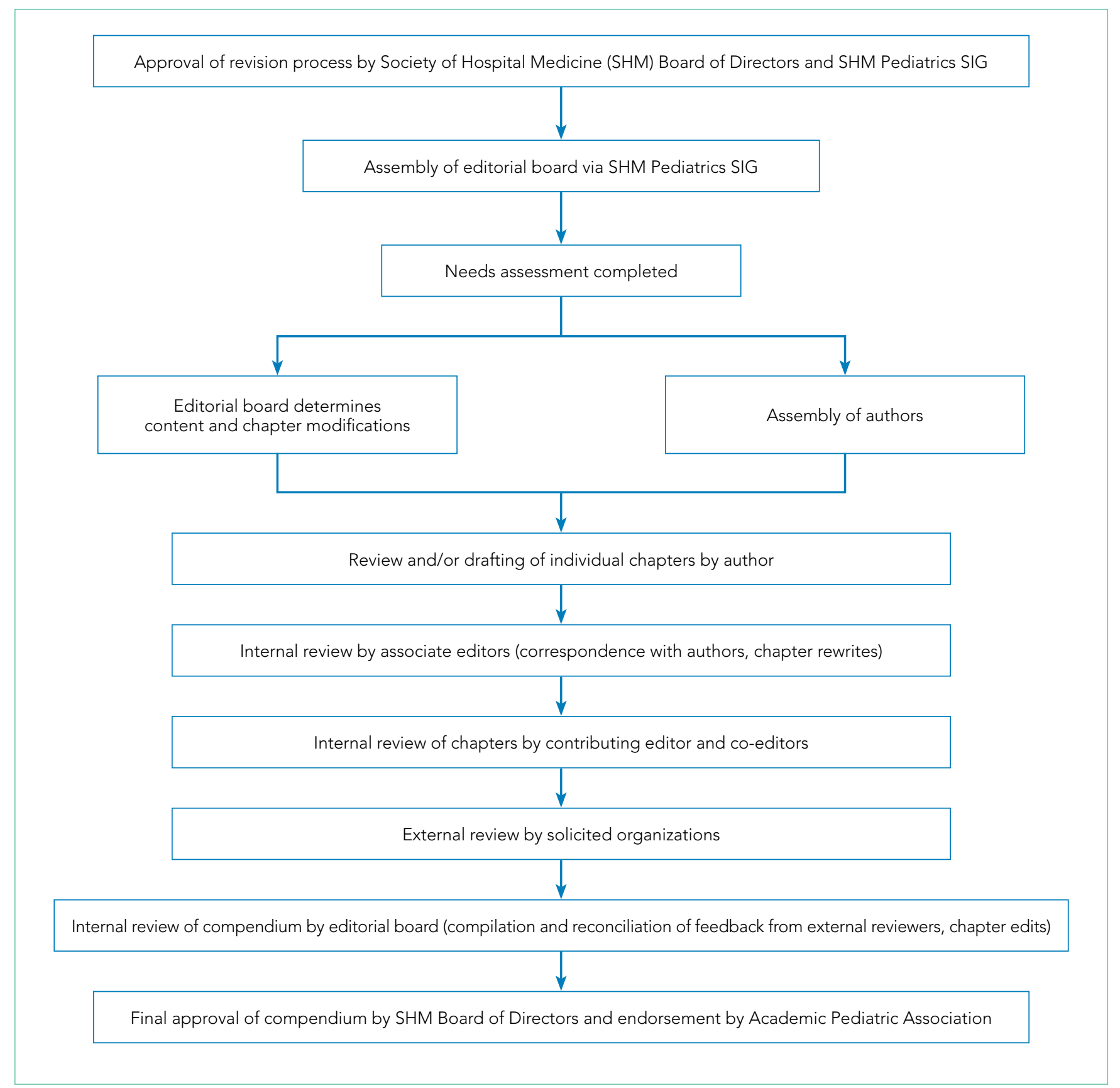

FIG. Process for The Pediatric Hospital Medicine Core Competencies: 2020 Revision

existing chapter or used to create a new chapter. There was a paucity of comments related to the deletion of chapters, and thus no chapters were entirely excluded. However, there were several comments supporting the exclusion of the suprapubic bladder tap procedure, and thus related content was eliminated from the relevant section in Core Skills. Of the 66 chapters in this revision, the needs assessment data directly informed the creation of 12 new chapters, as well as adjustments and/or additions to the titles of 7 chapters and the content of 29 chapters. In addition, the title of the Specialized Clinical Services section was changed to Specialized Services to represent that both clinical and nonclinical competencies reside in this sec- tion devoted to comprehensive management of these unique patient populations commonly encountered by pediatric hospitalists. Many of these changes are highlighted in Table 2.

\section{Author selection}

Authors from the initial work were invited to participate again as author of their given chapter. Subsequently, authors were identified for new chapters and chapters for which previous authors were no longer able to be engaged. Authors with content expertise were found by reviewing content from conferences, textbooks, and handbooks specific to the field of PHM. Any content expert who was not identified as a pediatric hospitalist 
TABLE 2. Substantive changes in The Pediatric Hospital Medicine Core Competencies: 2020 Revision

\section{New Chapters}

Common Clinical Diagnoses and Conditions

- Altered Mental Status

- Constipation

- Gastrointestinal and Digestive Disorders

- Neonatal Abstinence Syndrome/Neonatal Opioid Withdrawal Syndrome

Specialized Services

- Acute Behavioral and Psychiatric Conditions

- Adolescent and Young Adult Medicine

- Chronic Behavioral and Psychiatric Conditions

Core Skills

- Peri-procedural Care

- Preventive Care Services

Healthcare Systems: Supporting and Advancing Child Health

- Consultation and Co-management

- Family Centered Care

- Infection Control and Antimicrobial Stewardship

\section{Significant Chapter and Section Title Changes}

Common Clinical Diagnoses and Conditions

- Brief Resolved Unexplained Event (previously Apparent Life-Threatening Event)

- Head and Neck Disorders (previously Upper Airway Infections)

- Sepsis and Shock (previously Shock)

- Toxin Ingestion and Exposure (previously Toxic Ingestion)

Specialized Services (previously Specialized Clinical Services)

- Child with Medical Complexity (previously Technology-Dependent Children)

- Pediatric Interfacility Transport (previously Transport of the Critically III Child)

Core Skills

- Bladder Catheterization and Interpretation of Urinalysis (previously Bladder Catheterization/ Suprapubic Bladder Tap)

- Diagnostic Imaging (previously Radiographic Interpretation)

Healthcare Systems: Supporting and Advancing Child Health

- High Value Care (previously Cost-Effective Care)

- Handoffs and Transitions of Care (previously Transitions of Care)

- Leadership in Healthcare (previously Leading a Healthcare Team)

was paired with a pediatric hospitalist as coauthor. In addition, as with the editorial board, a deliberate effort was made to recruit a diverse author cohort, considering geographic location, primary work environment, time in practice, gender, and other factors.

The editorial board held numerous conference calls to review potential authors, and the SHM Pediatrics SIG was directly engaged to ensure authorship opportunities were extended broadly. This vetting process resulted in a robust author list and included members of all three of PHM's sponsoring societies in the United States. Once participation was confirmed, authors received an "author packet" detailing the process with the proposed timeline, resources related to writing learning objectives, the past chapter (if applicable), assigned associate editor, and other helpful resources.

\section{Internal and External Review Process}

After all chapters were drafted, the editorial board conducted a rigorous, internal review process. Each chapter was reviewed by at least one associate editor and two editors, with a focus on content, scope, and a standard approach to phrasing and formatting. In addition, the contributing editor reviewed all the chapters to ensure the community hospitalist perspective was adequately represented.

Thirty-two agencies and societies were solicited for external review, including both those involved in review of the previous edition and new stakeholder groups. External reviewers were first contacted to ascertain their interest in participating in the review process, and if interested, were provided with information on the review process. Robust feedback was received from the APA Hospital Medicine SIG, SHM Pediatrics and Medicine-Pediatrics SIGs, Association of Pediatric Program Directors Curriculum Committee, and 20 AAP committees, councils, and sections.

The feedback from the external reviewers and subsequent edits for each chapter were reviewed by at least one associate editor, two editors, and the contributing editor. Authors were engaged to address any salient changes recommended. As the final steps in the review process, the SHM Board of Directors approved the compendium and the APA provided their endorsement.

\section{SUMMARY AND FUTURE DIRECTIONS}

This second edition of The PHM Core Competencies: 2020 Revision addresses the knowledge, skills, attitudes, and systems organization and improvement objectives that define the field of pediatric hospital medicine and the leadership roles of pediatric hospitalists. This compendium reflects the recent changes in the practice and educational environments of pediatric hospitalists and can inform education, training, and career development for pediatric hospitalists across all environments in which comprehensive care is rendered for the hospitalized child. Future work at the local and national level can lead to development of associated curricula, conference content, and other training materials.

\section{Acknowledgments}

We wish to humbly and respectfully acknowledge the work of the authors, editors, and reviewers involved in the creation of the first edition, as well as this revision, of The PHM Core Competencies. In addition, we are grateful for the input of all pediatric hospitalists and other stakeholders who informed this compendium via contributions to the needs assessment survey, conference proceedings, publications, and other works. Finally, we acknowledge the support and work of SHM project coordinator, Nyla Nicholson, the SHM Pediatrics SIG, and the SHM Board of Directors.

Disclosures: SHM provided administrative support for project coordination ( $N$. Nicholson). No author, editor, or other involved member received any compensation for efforts related to this work. There are no reported conflicts of interest.

\section{References}

1. Pediatric hospital medicine core competencies. Stucky ER, Ottolini MC Maniscalco J, editors. J Hosp Med April 2010; Vol 5 No 2 (Supplement), 86 pages. Accessed August 7, 2019. https://www.journalofhospitalmedicine. com/jhospmed/issue/128018/journal-hospital-medicine-52

2. Association of American Medical Colleges: Analysis in Brief. Estimating the Number and Characteristics of Hospitalist Physicians in the United States and Their Possible Workforce Implications. August 2012 Edition. Accessed August 19, 2019. https://www.aamc.org/download/300620/data/aibvol12_ no3-hospitalist.pdf 
3. White CM, Thomson JE, Statile AM, et al. Development of a new care model for hospitalized children with medical complexity. Hosp Pediatr. 2017;7(7):410-414. https://doi.org/10.1542/hpeds.2016-0149

4. Committee on Hospital Care and Institute for Patient- and Family-Centered Care. Patient- and family-centered care and the pediatrician's role. Pediatr. 2012;129(2):394-404. https://doi.org/10.1542/peds.2011-3084

5. Pediatric Research in Inpatient Setting. Accessed August 27, 2019. https:// www.prisnetwork.org/

6. American Academy of Pediatrics. Value in Inpatient Pediatric Network. 2019 Edition. Accessed August 27, 2019. https://www.aap.org/en-us/professional-resources/quality-improvement/Pages/Value-in-Inpatient-Pediatrics.aspx.

7. American Academy of Pediatrics. Advancing Pediatric Educator Excellence Teaching Program. 2019 Edition. Accessed August 27, 2019. https://www. aap.org/en-us/continuing-medical-education/APEX/Pages/APEX.aspx

8. O'Toole JK, Starmer AJ, Calaman S, et al. I-PASS mentored implementation handoff curriculum: Champion training materials. MedEdPORTAL 2019;15:10794. https://doi.org/10.15766/mep 2374-8265.10794

9. Academic Pediatric Association. Pediatric Hospital Medicine 2018 Recap 2018 Edition. Accessed July 20, 2019. http://2018.phmmeeting.org/
10. PHM Fellowship Programs. 2019 Edition. Accessed Juyly 20, 2019. http:// phmfellows.org/phm-programs/

11. Shah NH, Rhim HJH, Maniscalco J, et al. The current state of pediatric hospital medicine fellowships: A survey of program directors. J Hosp Med. 2016;11:324-328.21. https://doi.org/10.1002/jhm.2571

12. Jerardi K, Fisher E, Rassbach $C$, et al. Development of a curricular framework for pediatric hospital medicine fellowships. Pediatr. 2017;140(1): e20170698.22. https://doi.org/10.1542/peds.2017-0698

13. Blankenburg R, Chase L, Maniscalco J, Ottolini M. Hospital Medicine Entrustable Professional Activities, American Board of Pediatrics, 2018. Accessed July 20, 2019. https://www.abp.org/subspecialty-epas\#Hospitalist\%20Medicine

14. Carraccio CL, Benson BJ, Nixon LJ, Derstine PL. From the educational bench to the clinical bedside: translating the Dreyfus Developmental Model to the learning of clinical skills. Accad Med. 2008;83(8):761-767. https://doi. org/10.1097/ACM.0b013e31817eb632

15. Nichani S, Crocker J, Fetterman N, Lukela M. Updating the core competencies in hospital medicine-2017 revision: Introduction and methodology. J Hosp Med. 2017;4;283-287. https://doi.org/10.12788/jhm.2715 